\title{
A new neoagarobiose-producing agarase from Vibrio sp. LA1
}

\author{
Bokun Lin ${ }^{\mathrm{a}, \mathrm{b}, \dagger, *}$, Yandan Zheng ${ }^{\mathrm{b}, \mathrm{c}, \dagger}$, Jingxiao Huang ${ }^{\mathrm{a}}$, Junkang Shang ${ }^{\mathrm{a}}$, Yuli Yu ${ }^{\mathrm{a}, \mathrm{b}}$, Zhong Hu${ }^{\mathrm{b}, *}$ \\ a Dongguan Key Laboratory of Environmental Medicine, School of Public Health, Guangdong Medical \\ University, Dongguan 523808 China \\ b Department of Biology, Shantou University, Shantou 515063 China \\ c Training \& Information Center, Jieyang Polytechnic, Jieyang 522000 China \\ *Corresponding authors, e-mail: bklin@gdmu.edu.cn, hzh@stu.edu.cn \\ $\uparrow$ These authors contributed equally to this work.
}

Received 23 Sep 2020

Accepted 15 Mar 2021

\begin{abstract}
An agarolytic bacterium was isolated from the sea coast of Shantou in China and identified as Vibrio sp. LA1. The agarase gene agaA was cloned from Vibrio sp. LA1 by using degenerate oligonucleotide-primed PCR and genome walking technique. Gene agaA consists of a 2913 bp open reading frame encoding 970 amino acids; and the predicted molecular mass and isoelectric point were $108 \mathrm{kDa}$ and 4.46, respectively. Based on the amino acid sequence similarity, the encoded protein (AgaA) of gene agaA should be an agarase of glycoside hydrolase family GH50 with a catalytic domain of glycoside hydrolase family GH42. Soluble expression of AgaA in Escherichia coli was obtained and investigated. The optimal temperature and $\mathrm{pH}$ for the activity of the purified recombinant agarase were $35^{\circ} \mathrm{C}$ and $\mathrm{pH} 6$, respectively. The reducing reagent $\beta$-mercaptoethanol could increase the activity of agarase AgaA by more than $80 \%$. AgaA showed exo-lytic activity on agarose degradation. It decomposed agarose to yield neoagarobiose as the sole product, which was different from the agarase Aga41A from Vibrio sp. CN41 that showed 95\% identities of amino acid sequence to agarase AgaA. With single end product, purification procedure is easier than that with multi-products. Therefore, agarase AgaA could be a useful tool for producing the bioactive neoagarobiose.
\end{abstract}

KEYWORDS: agarase, exo-lytic, neoagarobiose, agarose, genome walking

\section{INTRODUCTION}

Agar, a component of the cell wall of red algae, is a complex polysaccharide consisting of agarose and agaropectin. Agarose comprises D-galactose and 3,6-anhydro-L-galactose as monomeric units, which are linked by alternative $\alpha-1,3$ - or $\beta$-1,4-glycosidic bonds to form polymers [1]. Agarases are glycoside hydrolases (GH) that hydrolyze the $\alpha-1,3$ bond of agarose ( $\alpha$-agarase) to produce agarooligosaccharides (AOS), and hydrolyze the $\beta-1,4$ bond of agarose $(\beta$-agarase) to produce neoagarooligosaccharides (NAOS). Marine agar-degrading organisms produce agarases to utilize agar as a convenient carbon source. Therefore, most of the reported agarases were isolated from marine bacteria [2].

According to the homology of amino acid sequences, agarases have been classified into five glycoside hydrolase families: GH16, GH50, GH86, GH96, and GH118, in the Carbohydrate-Active EnZyme database (CAZy) [3]. Among these fam- ilies, family GH16 included the largest number of agarases which generate neoagrotetraose (NA4) and neoagarohexaose (NA6) as the main products of agarose degradation. In addition, most of the reported agarases were endo-lytic enzymes, while only a few agarases of the family GH50 displayed exo-lytic activity.

Several carbohydrate molecules and derivatives have been reported to exert beneficial biological activities, such as isomaltooligosaccharides [4] and extracellular polysaccharides [5] with prebiotic activity for food use; and arbutin, a glycosylated hydroquinone from plant extract, for medical use [6]. Similarly, NAOS possess diverse biological activities, such as moisturizing effects and whitening effects on skin $[7,8]$, anti-oxidant activities [9-11], prebiotic effects $[11,12]$, and potential treatment on type II diabetes [13]. It has been recognized that besides being mainly used in NAOS production, agarases can also be used in recovery of DNA from agarose gel $[14,15]$, protoplast preparation of from red alga 
$[16,17]$, and the screening of signal peptides [15]. All these applications rely on the search and the large-scale production of potent agarases.

The high application values of NAOS boost researches for novel and unique agarases. This study aims to provide an agarase gene cloning method, using degenerate oligonucleotide-primed PCR and genome walking technique, contributing to the growing agarase resource, and the characterization of a novel neoagarobiose- producing exolytic agarase from the agarolytic bacterium Vibrio sp. LA1.

\section{MATERIALS AND METHODS}

\section{Bacterial strains, plasmids and growth conditions}

The agarolytic strain LA1 was isolated from seawater samples in seaweed farming area of Shantou sea coast in Guangdong Province, China. Strain LA1 was propagated at $25^{\circ} \mathrm{C}$ in ZoBell 2216E medium. Escherichia coli DH5 $\alpha$ and E. coli BL21 (DE3) were used for routine cloning and protein expression, respectively. Vectors pMD19-T (TaKaRa Bio, China) and pET-32a (+) (Merck, Germany) were used for the cloning of agarase gene and preparation of recombinant plasmids, respectively. Agarose (Agarose LE) was purchased from MDbio Inc. (Qingdao, China). Unless otherwise stated, all chemicals used were of analytical grade or higher.

\section{Identification of strain LA1}

Genomic DNA of strain LA1 was isolated using a Genomic DNA Purification Kit (Guangzhou Dongsheng Biotech, China). The 16S rRNA gene was amplified from the chromosomal DNA by PCR with the universal primers of $27 \mathrm{~F}$ (forward primer) and 1492R (reverse primer) in Table S1. The PCR products were ligated with the T-A cloning vectors; and, then, the ligation mixtures were transformed into E. coli DH5 $\alpha$ cells. Subsequently, the recombinant plasmids were purified and sequenced. The $16 \mathrm{~S}$ rRNA gene sequence was compared with sequences in the GenBank database for primary identification.

\section{Cloning of agarase gene}

In order to obtain the agarase gene of strain LA1, a multiple sequence alignment of reported agarases was performed and found two conserved amino acid sequences of agarases of which the sequences were "DPWCVGYFVDNEM" and "VGAHWFQYIDSPITG", respectively. Degenerate primers, DENf and DGEr, (Table S1) were accordingly designed to these two conserved amino acid sequences using the online tool CODEHOP, respectively [18]. The conserved gene fragment of agarase was amplified by degenerate oligonucleotide-primed PCR. Subsequently, in order to obtain the complete structural gene, three rounds of genome-walking PCR (Genome Walker Kit, TaKaRa Bio, China) were run to amplify the upstream and downstream sequences of the conserved gene fragment. Specific primers of Lnsp1-3 (Table S1) were used to amplify the N-terminal sequence of the complete structural gene, while specific primers of Lcsp1-3 were used to amplify the C-terminal sequence. The PCR products were directly ligated to the vector PMD19-T for sequencing.

\section{Bioinformation analysis}

The nucleotide sequence of agarase gene and its deduced protein product were analyzed by different programs. The reading frames, theoretical isoelectric point $(\mathrm{pI})$, and molecular weight (MW) were predicted by using the DNAstar program. The signal peptide sequence of agarase gene was predicted using the SignalP server [19]. The alignments of protein sequences were conducted with the tblastn program (http://www.ncbi.nlm.nih.gov/BLAST). The prediction of protein domain was performed in the database of InterPro (http://www.ebi.ac.uk/ interpro).

\section{Expression of the agaA gene and purification of the recombinant AgaA}

To amplify the target gene, including the signal peptide, primers, EXPf and EXPr, (Table S1) were designed with restriction enzyme sites of Nde I and Xho I at the N-terminus and C-terminus of agaA, respectively. A poly 6-histidine residue was added to the C-terminus of the protein in order to purify the protein by affinity chromatography. The recombinant plasmid (pET-32a-agaA) constructed based on the expression vector pET-32a (+) was finally transformed into $E$. coli BL21 (DE3) cells. The recombinant $E$. coli BL21 (DE3) cells harboring the agarase gene were inoculated in LB medium and grown to mid-log phase at $37^{\circ} \mathrm{C}$, then they were induced for expression with isopropyl- $\beta$-thiogalactopyranoside (IPTG) to a final concentration of $0.5 \mathrm{mM}$, and incubated at $15^{\circ} \mathrm{C}$ for an additional $16 \mathrm{~h}$. The cells were collected by centrifugation, suspended in $20 \mathrm{ml}$ of $20 \mathrm{mM}$ Tris- $\mathrm{HCl}$ buffer (pH 8.0), and disrupted on ice by sonication. To maintain its bioactivity, the His-tagged agarase was purified with $\mathrm{Ni}^{2+}$ affinity column under native conditions according to the recommendations of the manufacturer 
(Beijing RuiDaHengHui Co., Ltd, China). The purity and homogeneity of the purified protein were analyzed by sodium dodecyl sulfate polyacrylamide gel electrophoresis (SDS-PAGE).

\section{Assay of agarase activity}

Agarase activity was determined by the enzymatic production of reducing sugars from agarose as described by Lin et al [20] with some modifications. The reaction mixture, containing $200 \mu \mathrm{l}$ appropriately diluted enzyme and $800 \mu \mathrm{l}$ of $0.25 \%$ agarose in $\mathrm{pH} 8$ Tris-HCl buffer $(20 \mathrm{mM})$ in a test tube, was incubated at $42{ }^{\circ} \mathrm{C}$ for $30 \mathrm{~min}$. After incubation, the sample was mixed with $2 \mathrm{ml}$ of 3,5-dinitrosalicylic acid (DNS) reagent and heated in boiling water for $15 \mathrm{~min}$. After cooling the test tube in a cold-water bath, the mixture was diluted to $10 \mathrm{ml}$ with deionized water. The absorbance at $540 \mathrm{~nm}$ was subsequently recorded. The amounts of reducing sugars generated were determined using D-galactose as a standard. One unit of the enzyme activity was defined as the amount of enzyme required to produce $1 \mu \mathrm{mol}$ reducing sugar per minute.

\section{Biochemical characterization of agarase AgaA}

The temperature effects on agarase activity were measured at different temperatures $\left(25-50^{\circ} \mathrm{C}\right)$ in $20 \mathrm{mM}$ Tris- $\mathrm{HCl}$ buffer ( $\mathrm{pH}$ 8). The $\mathrm{pH}$ effects on agarase activity were assayed at $35^{\circ} \mathrm{C}$ in a pH range of 4-11 using a combination of four different buffer systems: $50 \mathrm{mM}$ sodium citrate ( $\mathrm{pH} 4-6)$, $50 \mathrm{mM}$ Tris- $\mathrm{HCl}$ (pH 7-8), $50 \mathrm{mM}$ Tris-Glycine (pH 9-10), and $50 \mathrm{mM} \mathrm{Na}{ }_{2} \mathrm{HPO}_{4}-\mathrm{NaOH}(\mathrm{pH} \mathrm{11).}$ Effects of various cations and reagents on the enzyme reaction were assessed, including $\mathrm{KCl}, \mathrm{MgCl}_{2}$, $\mathrm{CaCl}_{2}$, ethylene diamine tetraacetic acid (EDTA), $\beta$ mercaptoethanol, and urea.

\section{Agarose degradation of agarase AgaA}

The products of agarose degradation were analyzed by thin layer chromatography (TLC). After mixing the enzyme solution with $0.2 \%$ agarose in $50 \mathrm{mM}$ sodium citrate buffer $(\mathrm{pH} 6)$ and incubated at $35^{\circ} \mathrm{C}$ for various time periods, the reaction mixtures were applied to a silica gel TLC plate along with the standards, which were neoagarobiose (NA2), neoagarotetraose (NA4), neoagarohexaose (NA6), and neoagarooctaose (NA8). An aliquot $(10 \mu \mathrm{l})$ of the reaction mixture was loaded on a silica gel 60 TLC plate (Shenghai Inc, Qingdao, China) and then developed with a solvent mixture composed of $n$ butanol/acetic acid/ $\mathrm{H}_{2} \mathrm{O}$ (2:1:1, by volume). The reaction products (NAOS) on the plate were visualized by spraying with $10 \%(\mathrm{v} / \mathrm{v}) \mathrm{H}_{2} \mathrm{SO}_{4}$, followed by heating at $85^{\circ} \mathrm{C}$. NAOS with different degrees of polymerization, which were prepared by other studied agarases in our laboratory and confirmed by mass spectrum as described by Lin et al [21], were used as standards and substrates to identify the cleavage pattern of AgaA.

\section{Nucleotide sequence accession numbers}

The 16S rRNA gene sequence of strain LA1 and agarase gene nucleotide sequence reported in this study had been submitted to the GenBank database under accession numbers JF682609 and JF461054, respectively.

\section{RESULTS}

\section{Identification of the agarolytic strain LA1}

The nucleotide sequence of the 16S rRNA gene (1512 bp) was aligned with sequences available in the GenBank database by using the BLASTN search program, and it exhibited maximum homology (99\%) with those of Vibrio species. The results indicated that the agarolytic strain LA1 was assigned to the genus Vibrio.

\section{Cloning and bioinformation analysis of the agarase gene}

A PCR product, about 700 bp long, was amplified by degenerate oligonucleotide-primed PCR sing degenerate primers DENf and DGEr). Subsequent sequencing and BLAST (NCBI) search showed that this gene fragment of $700 \mathrm{bp}$ was probably a partial sequence of agarase gene. The upstream and downstream sequences of the $700 \mathrm{bp}$ fragment were obtained by genome-walking PCR. The whole amplified sequence contained $4693 \mathrm{bp}$, in which a complete open reading frame (ORF) of $2913 \mathrm{bp}$ was found and named agaA. The gene agaA encodes a novel agarase with an initiation codon of GTG. The upstream sequence of gene agaA, including the putative promoter sequence and the ribosomebinding site, is shown in Fig. 1 . The -10 region was located 39 bp upstream from the initiation codon. The putative ribosome-binding site (SD sequence) was located $7 \mathrm{bp}$ upstream from the initiation codon. The encoded protein AgaA has a theoretical molecular mass of $108 \mathrm{kDa}$ and an isoelectric point $(\mathrm{p} I)$ of 4.46 . Analysis on the SignalP server revealed that the protein possesses a signal peptide of 23 amino acids (aa). And according to the analysis results in the database of InterPro, there 


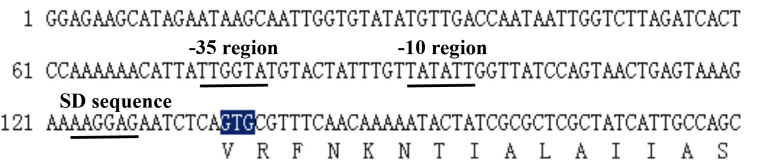

Fig. 1 The upstream sequence of gene agaA. The start codon (GTG) was in shadow. The -35 region, -10 region, and SD sequence were underlined. The putative ribosome-binding site (SD sequence) was located 7 bp upstream from the initiation codon.

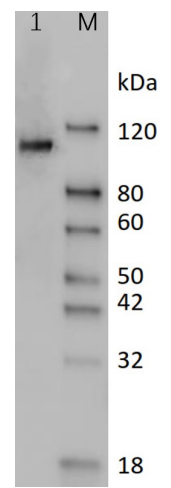

Fig. 2 SDS-PAGE of the recombinant AgaA overexpressed in $E$. coli. Lane M: protein markers; Lane 1: purified proteins by histidine-tag affinity chromatography.

was an N-terminal CBM-like domain (246-418 aa CBM: carbohydrate-binding module) and a catalytic domain of glycoside hydrolase family 42 (677-769 aa) in the encoded protein. In the conserved domain database of GenBank, the amino acid sequence of AgaA showed highest identities to reported agarases of glycoside hydrolase family GH50: $95 \%$ to agarase Aga41A of Vibrio sp. CN41 [22], 50\% to agarase HZ2 from Agarivorans sp. HZ105 [21], and 50\% to agarase AgaB from Vibrio sp. JT0107 [23]. Besides, the Carbohydrate-Active EnZyme database had assigned agarase AgaA to family GH50. Therefore, agarase AgaA should be a member of family GH50.

\section{Expression of $A g a A$ gene and purification of the recombinant AgaA}

The structural gene of AgaA was cloned into the pETHis expression system and conditionally expressed as a C-terminally His-tagged recombinant protein. When the transformed organism E. coli BL21 (DE3) harboring pET-32a-AgaA was inoculated in LB agar plate, there was no pits or clear zone-formingaround colonies. After IPTG induction at the temperature of $15^{\circ} \mathrm{C}$, agarase activity of the recombinant protein was detected in the supernatant of the

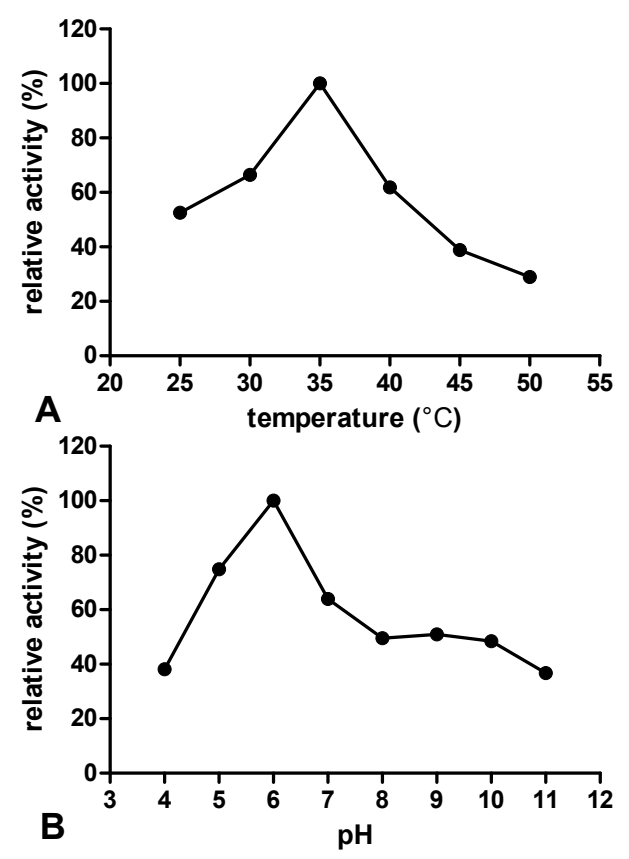

Fig. 3 Effects of temperature (A) and $\mathrm{pH}$ (B) on activity of AgaA. Temperature profiles were measured at different temperatures $\left(25-50^{\circ} \mathrm{C}\right)$ in $20 \mathrm{mM}$ Tris-HCl buffer ( $\left.\mathrm{pH} 8\right)$. The agarase activity determined at $35^{\circ} \mathrm{C}$ was $1.44 \mathrm{U} / \mathrm{mg}$, and it was set as $100 \%$ relative activity. $\mathrm{pH}$ profiles were assayed at $35^{\circ} \mathrm{C}$ in a $\mathrm{pH}$ range of 4-11 in which a combination of four different buffer systems was used: $50 \mathrm{mM}$ sodium citrate ( $\mathrm{pH} 5-6), 50 \mathrm{mM}$ Tris- $\mathrm{HCl}(\mathrm{pH} 7-$ 8), $50 \mathrm{mM}$ Tris-Glycine (pH 9-10), and $50 \mathrm{mM} \mathrm{Na}_{2} \mathrm{HPO}_{4}^{-}$ $\mathrm{NaOH}$ (pH11). The agarase activity determined at $\mathrm{pH} 6.0$ was $2.63 \mathrm{U} / \mathrm{mg}$, and it was set as $100 \%$ relative activity.

cell lysates. The recombinant protein was present mostly in a soluble form, instead of expressing as inclusion bodies. The observed molecular weight of recombinant AgaA was about $109 \mathrm{kDa}$ in the SDSPAGE including the 6-histidine tag, and it was in agreement with the theoretical molecular mass of the mature protein (Fig. 2).

\section{Biochemical characterization of agarase AgaA}

The optimum temperature of agarase activity of the recombinant AgaA was determined to be around $35^{\circ} \mathrm{C}$. And the agarase activity decreased sharply at temperatures higher than $45^{\circ} \mathrm{C}$. The optimum $\mathrm{pH}$ for the activity was 6.0 , but more than $50 \%$ of the maximal activity was retained over a broad range of pH 5-9 (Fig. 3). The results in Table 1 showed that the agarase activity of AgaA was conspicuously inhibited by $\mathrm{Ca}^{2+}$ ion as well as EDTA, especially at the concentration of $100 \mathrm{mM} . \mathrm{K}^{+}, \mathrm{Mg}^{2+}$, and urea 
Table 1 Effects of cations and reagents on AgaA activities. Standard enzyme assays were performed at $35^{\circ} \mathrm{C}$ in $50 \mathrm{mM}$ sodium citrate buffer $(\mathrm{pH} \mathrm{6)}$ supplemented with the tested metal ions and reagents at two different concentrations. The agarase activity of control without adding any chemical was $2.63 \mathrm{U} / \mathrm{mg}$, and it was set as $100 \%$ relative activity.

\begin{tabular}{lcc}
\hline \multirow{2}{*}{ Reagent } & \multicolumn{2}{c}{$\begin{array}{c}\text { Relative activity (\%) of AgaA } \\
\text { Reagent concentration }\end{array}$} \\
\cline { 2 - 3 } & $10 \mathrm{mM}$ & $100 \mathrm{mM}$ \\
\hline $\mathrm{KCl}$ & 109.3 & 96.1 \\
$\mathrm{MgCl}_{2}$ & 98.0 & 98.5 \\
$\mathrm{CaCl}_{2}$ & 83.9 & 14.6 \\
Urea & 95.7 & 93.8 \\
-mercaptoethanol $_{\text {EDTA }}$ & 189.2 & 182.0 \\
None & 85.7 & 0 \\
\hline
\end{tabular}

barely had negative effects on the agarase activity even at $100 \mathrm{mM}$. However, $\beta$-mercaptoethanol, a reducing reagent, was found to increase the activity of AgaA by more than $80 \%$ at both $10 \mathrm{mM}$ and $100 \mathrm{mM}$.

\section{Agarose degradation of agarase AgaA}

As shown in Fig. 4, the enzyme hydrolyzed agarose to generate NA2 as the sole product during incubation for 5-60 min without any other reaction intermediates, such as NA4 or NA6. As exo-agarases cleave dimers from the end of agarose chains to yield NA2; therefore, AgaA should be an exoagarase.

\section{DISCUSSION}

The N-terminal CBM-like domain found in agarase AgaA in this study has been described in the exoagarase Aga50D from the marine bacterium Saccharophagus degradans [24]. A loop in the CBM-like domain is involved in forming the roof of the active site channel. The contribution of the CBM-like domain to formation of the active site of the enzyme supports a role in substrate recognition explaining the exo-mode of beta-agarase action.

Agarases are generally classified in the CAZy database into five glycoside hydrolase families: GH16, GH50, GH86, GH96, and GH118. To date, a few agarases of family GH50 have been reported, and some are exo-agarases. The endo-agarases of family GH50 generally degrade agarose to yield NA4 and NA2 as the main products. For example, agarase Aga41A from Vibrio sp. CN41, which shows

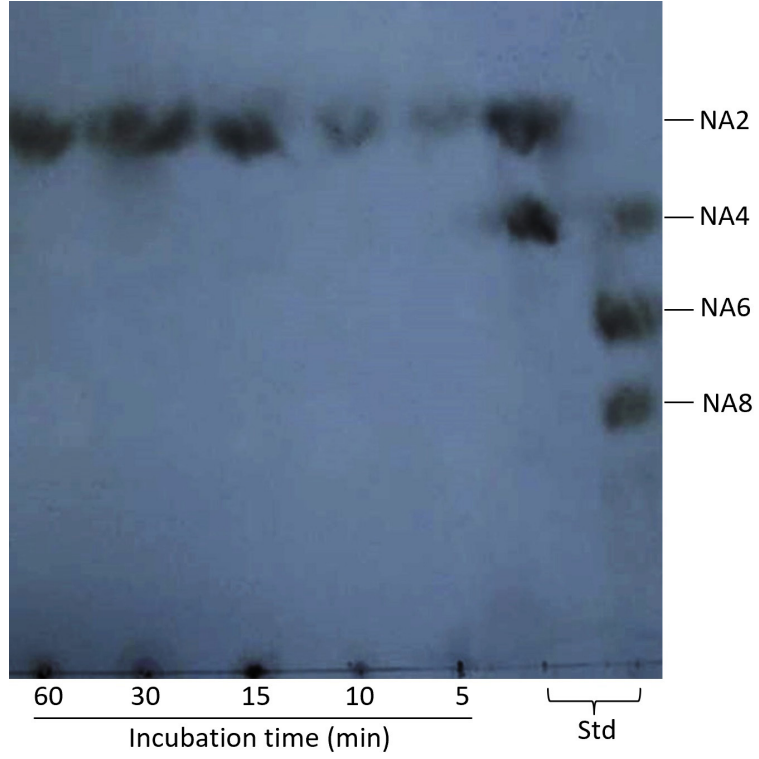

Fig. 4 TLC analysis of agarose degradation by agarase AgaA. Assays were performed in $80 \mu \mathrm{l}$ reaction mixtures containing $40 \mu \mathrm{l}$ of purified agarase and the equivalent volume of agarose substrate $(0.2 \%, \mathrm{w} / \mathrm{v}$, prepared in $50 \mathrm{mM}$ sodium citrate buffer $\mathrm{pH} 6)$ at $35^{\circ} \mathrm{C}$ for a series of incubation times: $5 \mathrm{~min}, 10 \mathrm{~min}, 15 \mathrm{~min}, 30 \mathrm{~min}$, and $60 \mathrm{~min}$. Neoagarobiose (NA2), neoagarotetraose (NA4), neoagarohexaose (NA6), and neoagarooctaose (NA8) were used as standards (Std).

95\% identities of amino acid sequence to agarase AgaA, decomposes agarose to yield NA4 as the end product [22]; and agarase HZ2 from Agarivorans sp. HZ105, which shows 50\% identities of amino acid sequence to agarase AgaA, produces NA4 as the main product [21]. However, the exo-agarases of family GH50 generally hydrolyze agarose to produce NA2 as the sole product, including the agarase AgaA in this study. Therefore, the agarase AgaA should be assigned to family GH50 based on amino acid similarity and the mode of agarose degradation. Besides, agarase AgaA also carries conserved amino acid sequences of family GH42, as distinct from other reported agarases.

In most cases, agarase activity is inhibited by $\beta$-mercaptoethanol ( $\beta$-Me) or dithiothreitol (DTT). However, the agarase activity of AgaA in this study was stimulated by $82-89 \%$ in the presence of $\beta$-Me, suggesting the possible existence of thiol in the catalytic site. The stimulatory effects of $\beta$-Me have also been displayed by several other agarases belonging to different glycoside hydrolase families, such as agarase HZ2 of family GH50 from Agarivorans sp. 
HZ105 [21], agarase AgaXa of family GH118 from Catenovulum sp. X3 [25], and $\beta$-agarase of family GH16 from Microbulbifer sp. Q7 [26]. This indicates that the stimulatory effect of $\beta$-Me on the agarases is not glycoside hydrolase family-dependent. The same phenomenon has also been found on the negative effects of $\mathrm{Ca}^{2+}$ on the activities of agarases that belong to different glycoside hydrolase families, such as agarase AgaA of family GH50 in this study, $\beta$-agarase Aga4436 of family GH16 from Flammeovirga sp. OC4 [27], and agarase AgaJ11 of family GH16 [28]. This suggests that the enzyme activities of those agarases do not require $\mathrm{Ca}^{2+}$, unlike the early reported agarases of family GH16 which have $\mathrm{Ca}^{2+}$ ion-binding sites and of which the enzyme activities are dependent upon the presence of $\mathrm{Ca}^{2+}$ [29]. The protein denaturant urea generally has negative effects on enzyme activity. However, in this study it showed that the agarase AgaA was resistant to $100 \mathrm{mM}$ urea. Actually, there were also some other reported agarases, of which the activities were not affected by urea. For example, agarase ID2563 could resist to $5 \mathrm{mM}$ urea [26] and agarases YM01-1 and YM01-3 were resistant to $10 \mathrm{mM}$ urea [30,31].

NA2 is a rare reagent showing both moisturizing and whitening effects with low cytotoxicity [32]. Nowadays, almost all agarases degrade agarose to yeild at least two end products, except the exoagarases of family GH50 hydrolyze agarose to produce NA2 as the sole product. With single end product, purification procedure is easier than that with multi-products. Therefore, agarase AgaA in this study could be a useful tool for producing the bioactive neoagarobiose.

In conclusion, the agarase gene agaA of 2913 bp was cloned from the agarolytic bacterium Vibrio sp. LA1. The encoded protein (AgaA) of gene agaA should be an agarase of glycoside hydrolase family GH50 with a catalytic domain of glycoside hydrolase family GH42. The optimal temperature and $\mathrm{pH}$ for the activity of the purified recombinant agarase were $35^{\circ} \mathrm{C}$ and $\mathrm{pH} 6$, respectively. AgaA showed exo-lytic activity on agarose degradation. It decomposed agarose to yield neoagarobiose (NA2) as the sole product.

\section{Appendix A. Supplementary data}

Supplementary data associated with this article can be found at http://dx.doi.org/10.2306/ scienceasia1513-1874.2021.045.
Acknowledgements: This work was supported by Funds for PHD researchers of Guangdong Medical University in 2017 (No. 2XB17026), Guangdong Yang Fan Project (4YF16003G), the National Natural Science Foundation of China (31200077) and the Discipline Construction Funds for Public Health and Preventive Medicine in Guangdong Medical University (4SG20004G and 4SG19046G).

\section{REFERENCES}

1. Araki C (1956) Structure of the agarose constituent of agar-agar. Bull Chem Soc Jpn 29, 543-544.

2. Park SH, Lee CR, Hong SK (2020) Implications of agar and agarase in industrial applications of sustainable marine biomass. Appl Microbiol Biotechnol 104, 2815-2832.

3. Lombard V, Golaconda RH, Drula E, Coutinho PM, Henrissat B (2014) The Carbohydrate-active enzymes database (CAZy) in 2013. Nucleic Acids Res 42, D490-D495.

4. Kaulpiboon J, Rudeekulthamrong P, Watanasatitarpa S, Ito K, Pongsawasdi P (2015) Synthesis of longchain isomaltooligosaccharides from tapioca starch and an in vitro investigation of their prebiotic properties. J Mol Catal B Enz 120, 127-135.

5. Chaiongkarn A, Dathong J, Phatvej W, Saman P, Kuancha C, Chatanon L, Moonmungmee S (2019): Characterization of prebiotics and their synergistic activities with Lactobacillus probiotics for $\beta$ glucuronidase reduction. ScienceAsia 45, 538-546.

6. Kaewduangdee S, Chaveerach A, Tanee T, Siripiyasing P, Sudmoon R (2020) Effect of dried ethanol extract of arbutin-containing leaves from Artocarpus on tyrosinase inhibition and postharvest preservation. ScienceAsia 46, 420-428.

7. Yun EJ, Lee S, Kim JH, Kim BB, Kim HT, Lee SH, Pelton JG, Kang NJ, et al (2013) Enzymatic production of 3,6-anhydro-L-galactose from agarose and its purification and in vitro skin whitening and antiinflammatory activities. Appl Microbiol Biotechnol 97, 2961-2970.

8. Kim JH, Yun EJ, Yu S, Kim KH, Kang NJ (2017) Different levels of skin whitening activity among 3,6-anhydro-l-galactose, agarooligosaccharides, and neoagarooligosaccharides. Mar Drugs 15, ID 321.

9. Jonnadula R, Imran M, Vashist P, Ghadi SC (2020) Production of agar-derived antioxidants and single cell detritus from Gracilaria corticata using agarase from Microbulbifer sp. CMC-5. Proc Natl Acad Sci India Sect B Biol Sci 90, 73-78.

10. Xu S, Kan J, Hu Z, Liu Y, Du H, Pang G, Cheong K (2018) Quantification of neoagaro-oligosaccharide production through enzymatic hydrolysis and its anti-oxidant activities. Molecules 23, E1354.

11. Zhang YH, Song XN, Lin Y, Xiao Q, Du XP, Chen YH, Xiao AF (2019) Antioxidant capacity and prebiotic effects of Gracilaria neoagaro oligosaccharides pre- 
pared by agarase hydrolysis. Int J Biol Macromol 137, 177-186.

12. Zhang X, Aweya JJ, Huang ZX, Kang ZY, Bai ZH, Li $\mathrm{KH}$, He XT, Liu Y, et al (2020) In vitro fermentation of Gracilaria lemaneiformis sulfated polysaccharides and its agaro-oligosaccharides by human fecal inocula and its impact on microbiota. Carbohydr Polym 234, ID 115894.

13. Lin $F$, Yang $D$, Huang $Y$, Zhao $Y$, Ye J, Xiao $M$ (2019) The potential of neoagaro-oligosaccharides as a treatment of type II diabetes in mice. Mar Drugs 17, ID 541.

14. Jung S, Lee CR, Chi WJ, Bae CH, Hong SK (2017) Biochemical characterization of a novel cold-adapted GH39 $\beta$-agarase, AgaJ9, from an agar-degrading marine bacterium Gayadomonas joobiniege G7. Appl Microbiol Biotechnol 101, 1965-1974.

15. Zhang W, Sun L (2007) Cloning, characterization, and molecular application of a beta-agarase gene from Vibrio sp. strain V134. Appl Enviro Microbio 73, 2825-2831.

16. Huddy SM, Meyers AE, Coyne VE (2013) Protoplast isolation optimization and regeneration of cell wall in Gracilaria gracilis (Gracilariales, Rhodophyta). J Appl Phycol 25, 433-443.

17. Chen H, Chen W, Shi J, Chen Z, Zhang Y (2019) Isolation and callus formation of Gracilariopsis bailiniae (Gracilariales, Rhodophyta) protoplasts. J Oceanol Limnol 36, 2268-2277.

18. Staheli JP, Boyce R, Kovarik D, Rose TM (2011) CODEHOP PCR and CODEHOP PCR primer design. Methods Mol Biol 687, 57-73.

19. Armenteros JJ, Tsirigos KD, Sonderby CK, Petersen TN, Winther O, Brunak S, Heijne GV, Nielsen HB (2019) SignalP 5.0 improves signal peptide predictions using deep neural networks. Nat Biotechnol 37, 420-423.

20. Lin B, Liu Y, Lu G, Zhao M, Hu Z (2017) An agarase of glycoside hydrolase family 16 from marine bacterium Aquimarina agarilytica ZC1. FEMS Microbiol Lett 364, fnx012.

21. Lin B, Lu G, Zheng Y, Xie W, Li S, Hu Z (2012) Gene cloning, expression and characterization of a neoagarotetraose-producing $\beta$-agarase from a marine bacterium Agarivorans sp. HZ105. World $J$ Microb Biot 28, 1691-1697.

22. Liao L, Xu XW, Jiang XW, Cao Y, Yi N, Huo YY,
Wu Y, Zhu X, etal (2011) Cloning, expression, and characterization of a new $\beta$-agarase from Vibrio sp. strain CN41. Appl Environ Microbiol 77, 7077-7079.

23. Sugano Y, Matsumoto T, Noma M (1994) Sequence analysis of the agaB gene encoding a new $\beta$-agarase from Vibrio sp. strain JT0107. Biochim Biophys Acta 17, 105-108.

24. Pluvinage B, Hehemann JH, Boraston AB (2013) Substrate recognition and hydrolysis by a family 50 exo- $\beta$-agarase, Aga50D, from the marine bacterium Saccharophagus degradans. J Biol Chem 288, 28078-28088.

25. Xie W, Lin B, Zhou Z, Lu G, Lun J, Xia C, Li S, Hu Z (2013) Characterization of a novel $\beta$-agarase from an agar-degrading bacterium Catenovulum sp. X3. Appl Microbiol Biotechnol 97, 4907-4915.

26. Su Q, Jin T, Yu Y, Yang M, Mou H, Li L (2017) Extracellular expression of a novel $\beta$-agarase from Microbulbifer sp. Q7, isolated from the gut of sea cucumber. AMB Express 7, ID 220.

27. Chen X, Hou Y, Jin M, Zeng R, Lin H (2016) Expression and characterization of a novel thermostable and $\mathrm{pH}$-stable $\beta$-Agarase from deep-sea bacterium Flammeovirga sp. OC4. J Agr Food Chem 64, 7251-7258.

28. Jung S, Jeong B, Hong S, Lee C (2017) Cloning, expression, and biochemical characterization of a novel acidic GH16 $\beta$-agarase, AgaJ11, from Gayadomonas joobiniege G7. Appl Biochem Biotech 181, 961-971.

29. Allouch J, Jam M, Helbert W, Barbeyron T, Kloareg B, Henrissat B, Czjzek M (2003) The three-dimensional structures of two $\beta$-agarases. J Biol Chem 278, 47171-47180.

30. An K, Shi X, Cui F, Cheng J, Liu N, Zhao X, Zhang $\mathrm{XH}$ (2018) Characterization and overexpression of a glycosyl hydrolase family 16 beta-agarase YM011 from marine bacterium Catenovulum agarivorans YM01 $^{T}$. Protein Expr Purif 143, 1-8.

31. Cui F, Dong S, Shi X, Zhao X, Zhang XH (2014) Overexpression and characterization of a novel thermo stable $\beta$-Agarase YM01-3, from marine bacterium

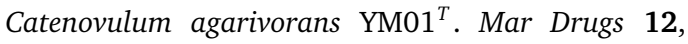
2731-2747.

32. Kobayashi R, Takisada M, Suzuki T, Kirimura K, Usami S (1997) Neoagarobiose as a novel moisturizer with whitening effect. Biosci Biotechnol Biochem 61, 162-163. 


\section{Appendix A. Supplementary data}

Table S1 Primer sequences.

\begin{tabular}{ll}
\hline Primer & Sequence $\left(5^{\prime}-3^{\prime}\right)$ \\
\hline $27 \mathrm{~F}$ & AGAGTTTGATCCTGGCTCAG \\
$1492 \mathrm{R}$ & TACGGCTACCTTGTTACGACTT \\
DENf & CAACTATGGATAAAAATTATTTAGTAGGTTTAGTAGATvarttyggnca \\
DGEr & AGTATCAGTTACAGATACAAATCCTACATTATAAttytcnccrtc \\
Lnsp1 & GCTACCTTCATCTCAATGCGGACTTC \\
Lnsp2 & ATAAGAACCCGACTCTGGGCGAAAC \\
Lnsp3 & GGTGTGTGTGTTGGCGGTTTTTGATA \\
Lcsp1 & CAGCAGCTCGCTATGTTGATGTGATG \\
Lcsp2 & GGTTTGTTCCACCCTGGCATTATCA \\
Lcsp3 & CATGGATTCACCAGTAACAGGGCGT \\
EXPf & GGGGGGTCCATATGCGTTTCAACAAAAATAC \\
EXPr & CCGCTCGAGATTCAAAGAACCAAA \\
\hline
\end{tabular}

